\title{
A LOTKA-VOLTERRA PREDATOR-PREY SYSTEM INVOLVING TWO PREDATORS
}

\author{
George Seifert
}

\begin{abstract}
We consider positive solutions of certain Lotka-Volterra systems consisting of three first-order differential equations which can model the population sizes of a prey and two predators where there also may be interaction between the predators. Under certain conditions on the interaction rates, one of the predators dies out while the remaining predator and prey approach periodic behavior as time increases. For a critical case, each positive solution of the system will be periodic.
\end{abstract}

Consider the system of ordinary differential equations

$$
\begin{aligned}
& x^{\prime}=a x-x y-d x z \\
& y^{\prime}=-b y+x y+e y z \\
& z^{\prime}=-c z+d x z-e y z
\end{aligned}
$$

where $a, b, c$, and $d$ are positive constants and $e$ is a nonnegative constant. We will be concerned only with solutions $(x(t), y(t), z(t))$ such that $x(0), y(0), z(0)$ are all positive. It is easy to show that for such solutions, $x(t), y(t), z(t)$ are all positive for all $t \geq 0$; we omit the details.

In the special case $e=0$, this system could model a biological system consisting of two predators and a prey, whose population sizes are $y(t), z(t)$, and $x(t)$, respectively. We make no claim as to the biological accuracy of this model; we believe, however, that our results and methods are to some extent new and mathematically interesting.

It is easy to see that, in this case, (1) has critical points (i.e., equilibria) at $(0,0,0)$, $(b, a, 0)$, and $(c / d, 0, a / d)$. Further, it will follow from our theorem that if $c>d b$, all solutions $(x(t), y(t), z(t))$ satisfy $\left(x(t)-x_{0}(t)\right)^{2}+\left(y(t)-y_{0}(t)\right)^{2}+z^{2}(t) \rightarrow 0$ as $t \rightarrow \infty$, where $\left(x_{0}(t), y_{0}(t)\right)$ is periodic; in fact, if $x(0)=b$ and $y(0)=a$ then $\left(x_{0}(t), y_{0}(t)\right)=$ $(b, a)$ for all $t \geq 0$. For the predator-prey model in this case, we conclude that the $z$ predator dies out, i.e., fails to persist.

If $c<d b$, it will follow that any solution $(x(t), y(t), z(t))$ satisfies

$$
\left(x(t)-x_{0}(t)\right)^{2}+y^{2}(t)+\left(z(t)-z_{0}(t)\right)^{2} \rightarrow 0 \text { as } t \rightarrow \infty,
$$

where $\left(x_{0}(t), z_{0}(t)\right)$ is periodic; if $x(0)=c / d$ and $z(0)=a / d$, then $\left(x_{0}(t), z_{0}(t)\right)=$ $(c / d, a / d)$ for $t \geq 0$. In this case, the $y$-predator fails to persist.

Some of these results for these special cases of (1) have already been established; cf. $[3,4]$. However, the fact that, in each case, all solutions are asymptotically periodic apparently was not observed in these papers.

Received August 3, 1994, revised February 24, 1995.

1991 Mathematics Subject Classification. 34C25, 34D05, 92D25.

Key words and phrases: Lotka-Volterra predator-prey system. 
The purpose of this note is to examine the more general case where $e \geq 0$, i.e., cases where the predators can interact in the sense that the $y$-predator preys on the $z$-predator.

A critical point of (1) is said to be reachable if it is in the set $\{(x, y, z): x \geq 0, y \geq$ $0, z \geq 0\}$, and is said to be stable if it is stable in the usual sense of Liapunov; cf. [5], for example.

Theorem 1. Suppose $e a+c \neq d b$. Then the only possibly stable critical points of $(1)$ are $(c / d, 0, a / d)$ and $(b, a, 0)$. If ea $+c<d b$, all (positive) solutions $(x(t), y(t), z(t))$ satisfy $\left(x(t)-x_{0}(t)\right)^{2}+y^{2}(t)+\left(z(t)-z_{0}(t)\right)^{2} \rightarrow 0$ as $t \rightarrow \infty$ where $\left(x_{0}(t), z_{0}(t)\right)$ is periodic. If ea $+c>d b$, all solutions $(x(t), y(t), z(t))$ satisfy

$$
\left(x(t)-x_{0}(t)\right)^{2}+\left(y(t)-y_{0}(t)\right)^{2}+z^{2}(t) \rightarrow 0 \text { as } t \rightarrow \infty
$$

where $\left(x_{0}(t), y_{0}(t)\right)$ is periodic. If ea $+c=d b$, all solutions are periodic; in this case, the line segment in $(x, y, z)$ space defined by $y+d z=a, x+e z=b, x \geq 0, y \geq 0$, $z \geq 0$ consists of critical points of (1) and these are the only critical points with all coordinates nonnegative.

Proof. Case 1: $e a+c<d b$.

Let $x_{0}=c / d, y_{0}=0, z_{0}=a / d$, and $(x(t), y(t), z(t))$ be a solution of $(1)$. Put

$$
x_{1}(t)=x(t)-x_{0}, \quad x_{2}(t)=y(t)-y_{0}, \quad x_{3}(t)=z(t)-z_{0},
$$

and (1) becomes

$$
\begin{aligned}
& x_{1}^{\prime}=-\left(d x_{3}+x_{2}\right)\left(x_{1}+x_{0}\right), \\
& x_{2}^{\prime}=\left(x_{1}+e x_{3}\right) x_{2}+[(c+a e) / d-b] x_{2}, \\
& x_{3}^{\prime}=\left(d x_{1}-e x_{2}\right)\left(x_{3}+z_{0}\right) .
\end{aligned}
$$

It follows easily from the linearization of $(3)$ that $\left(x_{0}, y_{0}, z_{0}\right)$ is stable. So if

$$
\begin{aligned}
& F\left(x_{1}\right)=x_{1}-x_{0} \log \left(x_{1}+x_{0}\right), \\
& H\left(x_{3}\right)=x_{3}-z_{0} \log \left(x_{3}+z_{0}\right),
\end{aligned}
$$

and $V\left(x_{1}, x_{2}, x_{3}\right)=F\left(x_{1}\right)+x_{2}+H\left(x_{3}\right)$, then it follows from (3) that

$$
\frac{d}{d t} V\left(x_{1}(t), x_{2}(t), x_{3}(t)\right)=\left(\frac{c+e a}{d}-b\right) x_{2}(t) .
$$

So $x_{1}(t), x_{2}(t)$, and $x_{3}(t)$ are bounded above for $t \geq 0$. Since also $x_{1}(t)>-x_{0}$, $x_{2}(t)>0$, and $x_{3}(t)>-z_{0}$, it follows that $\left|x_{2}^{\prime}(t)\right|$ is bounded for $t \geq 0$, and so, by a simple argument using (4), that $x_{2}(t) \rightarrow 0$ as $t \rightarrow \infty$; we omit the details. Finally, since $V\left(x_{1}, x_{2}, x_{3}\right) \geq-x_{0} \log x_{0}-z_{0} \log z_{0}$ for $x_{1}>-x_{0}, x_{2}>0, x_{3}>-z_{0}$, we conclude that

$$
V\left(x_{1}(t), x_{2}(t), x_{3}(t)\right) \rightarrow L_{0} \text { as } t \rightarrow \infty, \text { where } m_{0} \leq L_{0}<\infty,
$$

and $m_{0}=-x_{0} \log x_{0}-z_{0} \log z_{0}$.

It can be shown that the set $\Gamma_{0}$ in $\left(x_{1}, x_{2}, x_{3}\right)$ space given by $\Gamma_{0} \equiv\left\{\left(x_{1}, 0, x_{3}\right)\right.$ : $\left.F\left(x_{1}\right)+H\left(x_{3}\right)=L_{0}\right\}$ is a simple closed curve if $L_{0}>m_{0}$ and the point $(0,0,0)$ if $L_{0}=m_{0}$. Since

$$
V(t)-\left(F\left(x_{1}(t)\right)+H\left(x_{3}(t)\right)\right) \rightarrow 0 \text { as } t \rightarrow \infty
$$


where $V(t)=V\left(x_{1}(t), x_{2}(t), x_{3}(t)\right)$, it follows that the $\omega$-limit set of $\left(x_{1}(t), x_{2}(t), x_{3}(t)\right)$ is a subset of $\Gamma_{0}$. Since this $\omega$-limit set is invariant for solutions of $(3)$, if $\left(\bar{x}_{1}, 0, \bar{x}_{3}\right) \in$ $\Gamma_{0}$, then the orbit of the solution $\left(\bar{x}_{1}(t), 0, \bar{x}_{3}(t)\right)$ of $(3)$ satisfying $\bar{x}_{1}(0)=\bar{x}_{1}, \bar{x}_{3}(0)=$ $\overline{x_{3}}, \bar{x}_{2}(0)=0$ lies in $\Gamma_{0}$. Assume first that $L_{0}>m_{0}$, and let $T>0$ be the least period of $\left(\bar{x}_{1},(t), \bar{x}_{3}(t)\right)$.

Since it can be shown that this solution $\left(\bar{x}_{1}(t), 0, \bar{x}_{3}(t)\right)$ is periodic, its orbit must coincide with $\Gamma_{0}$.

We show that for some $\tau, 0 \leq \tau \leq T,\left(x_{1}(t)-\bar{x}_{1}(t+\tau)\right)^{2}+x_{2}^{2}(t)+\left(x_{3}(t)-\bar{x}_{3}(t+\tau)\right)^{2}$ $\rightarrow 0$ as $t \rightarrow \infty$. To do this, we use the following notation and lemma. Define

$$
\begin{aligned}
v(t) & =\left(x_{1}(t), x_{2}(t), x_{3}(t)\right), \\
\bar{v}(t) & =\left(\bar{x}_{1}(t), 0, \bar{x}_{3}(t)\right), \\
u(t, \tau) & =|v(t)-\bar{v}(t+\tau)| .
\end{aligned}
$$

Here and henceforth, $|\cdot|$ denotes the Euclidean norm in $\mathbf{R}^{3}$.

Lemma. $\varlimsup_{t \rightarrow \infty} u(t, \tau) \equiv s(\tau)$ is continuous for $0 \leq \tau \leq T$.

Proof of Lemma. Clearly, $s(\tau)<\infty$ for $0 \leq \tau \leq T$. Since

$$
|s(\tau+\delta)-s(\tau)| \leq \varlimsup_{t \rightarrow \infty}|u(t, \tau+\delta)-u(t, \tau)|
$$

and

$$
\begin{aligned}
|u(t, \tau+\delta)-u(t, \tau)| & =|| v(t)-\bar{v}(t+\tau+\delta)|-| v(t)-\bar{v}(t+\tau)|| \\
& \leq|\bar{v}(t+\tau+\delta)-\bar{v}(t+\tau)|
\end{aligned}
$$

it follows from (5) that

$$
|s(\tau+\delta)-s(\tau)| \leq \varlimsup_{t \rightarrow \infty}|\bar{v}(t+\tau+\delta)-\bar{v}(t+\tau)| .
$$

Since $\bar{v}(t)$ is continuous and periodic, it follows that for a given $\epsilon>0$, there exists a $\delta(\epsilon)>0$ such that $|\bar{v}(t+\tau+\delta)-\bar{v}(t+\tau)|<\epsilon$ for $|\delta|<\delta(\epsilon), t \geq 0,0 \leq \tau \leq T$. Using (6), our lemma follows.

Now suppose there is no $\tau, 0 \leq \tau \leq T$, such that $v(t)-\bar{v}(t+\tau) \rightarrow 0$ as $t \rightarrow \infty$. Then for each $\tau, 0 \leq \tau \leq T$, we have $u(t, \tau) \nrightarrow 0$ as $t \rightarrow \infty$. So for each such $\tau$, we have $\varlimsup_{t \rightarrow \infty} u(t, \tau)=s(\tau)>0$. Using our lemma, we have

$$
\varlimsup_{t \rightarrow \infty} u(t, \tau) \geq \epsilon_{0} \quad \text { for some } \epsilon_{0}>0 \text { and } 0 \leq \tau \leq T \text {. }
$$

Since $v(t)$ approaches the orbit of $\bar{v}(t)$ as $t \rightarrow \infty$ and $\bar{v}(t)$ is $T$-periodic, for each $t>0$ there exists a $\tau(t), 0 \leq \tau(t)<T$, such that

$$
|v(t)-\bar{v}(t+\tau(t))| \rightarrow 0 \text { as } t \rightarrow \infty,
$$

i.e., take $\tau(t)$ such that

$$
|v(t)-\bar{v}(t+\tau(t))|=\min _{0 \leq \tau<T}|v(t)-\bar{v}(t+\tau)| .
$$

In fact, because of the convexity of the orbit of $\bar{v}(t)$, it follows that $\tau(t)$ is unique for $t$ large enough.

So there exists a $t_{1}>0$ such that

$$
u(t, \tau(t))=|v(t)-\bar{v}(t+\tau(t))|<\frac{\epsilon_{0}}{2} \text { for } t \geq t_{1} .
$$


But from (7) there exists a $t_{2}>t_{1}$ such that

$$
u\left(t_{2}, \tau\right)>\epsilon_{0} / 2 \quad \text { for all } \tau, \quad 0 \leq \tau \leq T,
$$

and, for $\tau=\tau\left(t_{2}\right)$, this contradicts (8). So there must be some $\tau, 0 \leq \tau \leq T$, such that

$$
|v(t)-\bar{v}(t+\tau)| \rightarrow 0 \text { as } t \rightarrow \infty \text {. }
$$

This completes the proof of Case 1 for $L_{0}>m_{0}$ since we may take

$$
\begin{aligned}
& x_{0}(t)=\bar{x}_{1}(t+\tau)+x_{0}, \\
& y_{0}(t)=0, \\
& z_{0}(t)=\bar{x}_{3}(t+\tau)+z_{0} .
\end{aligned}
$$

If $L_{0}=m_{0}$, then $\Gamma_{0}=(0,0,0)$, and it follows easily that $\left(x_{1}(t), x_{2}(t), x_{3}(t)\right) \rightarrow$ $(0,0,0)$ as $t \rightarrow \infty$; we omit the details.

Case 2: $e a+c>d b$.

We use the same argument as in Case 1 except that we define $x_{1}(t)=x(t)-b$, $x_{2}(t)=y(t)-a, x_{3}(t)=z(t)$ and use

$$
V\left(x_{1}, x_{2}, x_{3}\right)=x_{1}-b \log \left(x_{1}+b\right)+x_{2}-a \log \left(x_{2}+a\right)+x_{3}
$$

in place of the $V$ of Case 1 . Thus we arrive at the conclusion that $x_{3}(t) \rightarrow 0$ as $t \rightarrow \infty$ and that (2) holds; we omit the details.

Case 3: $e a+c=d b$.

It is easy to show that the critical points of (1) consist of the line in $(x, y, z)$ space defined by $y+d z=a, x+e z=b$. We shall be concerned with the segment of this line defined by $x \geq 0, y \geq 0, z \geq 0$.

Let $\left(x_{0}, y_{0}, z_{0}\right)$ be an interior point of this line segment. Let $(x(t), y(t), z(t))$ be a solution of $(1)$ with $(x(0), y(0), z(0))$ not a critical point of $(1)$, and such that $x(0)>0$, $y(0)>0, z(0)>0$. Put

$$
\begin{aligned}
& x_{1}(t)=x(t)-x_{0} \\
& x_{2}(t)=y(t)-y_{0}, \\
& x_{3}(t)=z(t)-z_{0} .
\end{aligned}
$$

Then (1) becomes

$$
\begin{aligned}
& x_{1}^{\prime}=-\left(x_{2}+d x_{3}\right)\left(x_{1}+x_{0}\right), \\
& x_{2}^{\prime}=\left(x_{1}+e x_{3}\right)\left(x_{2}+y_{0}\right), \\
& x_{3}^{\prime}=\left(d x_{1}-e x_{2}\right)\left(x_{3}+z_{0}\right) .
\end{aligned}
$$

If

$$
\begin{aligned}
& F\left(x_{1}\right)=x_{1}-x_{0} \log \left(x_{1}+x_{0}\right), \\
& G\left(x_{2}\right)=x_{2}-y_{0} \log \left(x_{2}+y_{0}\right), \\
& H\left(x_{3}\right)=x_{3}-z_{0} \log \left(x_{3}+z_{0}\right),
\end{aligned}
$$

and $V\left(x_{1}, x_{2}, x_{3}\right)=F\left(x_{1}\right)+G\left(x_{2}\right)+H\left(x_{3}\right)$, it follows, using (9), that

$$
\frac{d}{d t} V\left(x_{0}(t), x_{2}(t), x_{3}(t)\right)=0 \text { for } t \geq 0
$$


and so

$$
V\left(x_{1}(t), x_{2}(t), x_{3}(t)\right)=c_{0}=V\left(x_{1}(0), x_{2}(0), x_{3}(0)\right), \quad t \geq 0 .
$$

Note that $c_{0} \geq-x_{0} \log x_{0}-y_{0} \log y_{0}-z_{0} \log z_{0}$ since the right side of this last inequality is the minimum value of $V\left(x_{1}, x_{2}, x_{3}\right)$ for $x_{1}>-x_{0}, x_{2}>-y_{0}, x_{3}>-z_{0}$, which is $V(0,0,0)$.

Since $\left(x_{1}(0), x_{2}(0), x_{3}(0)\right) \neq(0,0,0)$,

$$
V\left(x_{1}, x_{2}, x_{3}\right)=c_{0}
$$

defines a closed bounded strictly convex 2 -surface, strictly convex in the sense that any line in $\left(x_{1}, x_{2}, x_{3}\right)$ space intersects it in at most 2 points. To show this, consider such a line given, say, by

$$
\left\{\left(a_{1} t+b_{1}, a_{2} t+b_{2}, a_{3} t+b_{3}\right): t \in R\right\}
$$

where $a_{1}^{2}+a_{2}^{2}+a_{3}^{2}>0$. Define $M(t)=V\left(a_{1} t+b_{1}, a_{2} t+b_{2}, a_{3} t+b_{3}\right)$. It is easy to see that $M^{\prime \prime}(t)>0$ for all $t$, and so $M(t)=c_{0}$ can have at most 2 solutions.

From (10), we see that the orbit of $\left(x_{1}(t), x_{2}(t), x_{3}(t)\right)$ lies on that surface. If we consider the image of this surface under the change of variables

$$
x_{1}=x-x_{0}, \quad x_{2}=y-y_{0}, \quad x_{3}=z-z_{0},
$$

say $S$, given by

$$
V\left(x-x_{0}, y-y_{0}, z-z_{0}\right)=c_{0},
$$

we conclude that $(x(t), y(t), z(t)) \in S$ for $t \geq 0$.

Let $\left(\bar{x}_{0}, \bar{y}_{0}, \bar{z}_{0}\right)$ be another critical point of $(1)$ distinct from $\left(x_{0}, y_{0}, z_{0}\right)$ and such that $\bar{x}_{0}>0, \bar{y}_{0}>0, \bar{z}_{0}>0$. Then, proceeding as above with $\left(x_{0}, y_{0}, z_{0}\right)$ replaced by $\left(\bar{x}_{0}, \bar{y}_{0}, \bar{z}_{0}\right)$, we find that the solution $(x(t), y(t), z(t))$ also satisfies $(x(t), y(t), z(t)) \in \bar{S}$ for $t \geq 0$, where $\bar{S}$ is the 2 -surface in $(x, y, z)$ space defined by

$$
\begin{gathered}
V\left(x-\bar{x}_{0}, y-\bar{y}_{0}, z-\bar{z}_{0}\right)=\bar{c}_{0}, \\
\bar{c}_{0}=V\left(x(0)-\bar{x}_{0}, y(0)-\bar{y}_{0}, z(0)-\bar{z}_{0}\right) ;
\end{gathered}
$$

here $\bar{S}$ also is bounded, closed, and strictly convex. So $(x(t), y(t), z(t)) \in S \cap \bar{S}$ for $t \geq 0$.

We show next that $S \cap \bar{S}$ is a simple closed curve. Let $\left(x_{1}, y_{1}, z_{1}\right) \in S \cap \bar{S}$. We will show later that $\left(x_{1}, y_{1}, z_{1}\right)$ cannot be a critical point of (1). Calculating the gradients of $V\left(x-x_{0}, y-y_{0}, z-z_{0}\right)$ and $V\left(x-\bar{x}_{0}, y-\bar{y}_{0}, z-\bar{z}_{0}\right)$ at $\left(x_{1}, y_{1}, z_{1}\right)$, we get, respectively,

and

$$
\left(1-x_{0} / x_{1}, 1-y_{0} / y_{1}, 1-z_{0} / z_{1}\right)
$$

$$
\left(1-\bar{x}_{0} / x_{1}, 1-\bar{y}_{0} / y_{1}, 1-\bar{z}_{0} / z_{1}\right) .
$$

Using the facts that $\left(x_{0}, y_{0}, z_{0}\right)$ and $\left(\bar{x}_{0}, \bar{y}_{0}, \bar{z}_{0}\right)$, being distinct critical points, satisfy each of the equations $x=b-e z$ and $y=a-d z$ and that $\left(x_{1}, y_{1}, z_{1}\right)$ is not a critical point, i.e., does not satisfy one of these two equations, one can show, after detailed computations which we omit, that the cross product of these gradients is non-zero. This shows that $S \cap \bar{S}$ is a simple closed curve. 
We now show that $S \cap \bar{S}$ cannot contain a critical point of (1). Suppose $(x, y, z)$ is a critical point in $S \cap \bar{S}$. Then $(x, y, z) \in S$ implies $V\left(x-x_{0}, y-y_{0}, z-z_{0}\right)=$ $V\left(x(0)-x_{0}, y(0)-y_{0}, z(0)-z_{0}\right)$, from which we get

$$
x-x(0)+y-y(0)+z-z(0)-x_{0} \log \frac{x}{x(0)}-y_{0} \log \frac{y}{y(0)}-z_{0} \log \frac{z}{z(0)}=0 .
$$

Similarly, $(x, y, z) \in \bar{S}$ implies

$$
x-x(0)+y-y(0)+z-z(0)-\bar{x}_{0} \log \frac{x}{x(0)}-\bar{y}_{0} \log \frac{y}{y(0)}-\bar{z}_{0} \log \frac{z}{z(0)}=0 .
$$

Using the last 2 equations we get

$$
\left(x_{0}-\bar{x}_{0}\right) \log \frac{x}{x(0)}+\left(z_{0}-\bar{z}_{0}\right) \log \frac{y}{y(0)}+\left(z_{0}-\bar{z}_{0}\right) \log \frac{z}{z(0)}=0 .
$$

Using the fact that $(x, y, z)$ is a critical point of (1), we have $x=b-e z$ and $y=a-d z$. So, using (11), we get

$$
\left(x_{0}-\bar{x}_{0}\right) \log \frac{(b-e v)}{x(0)}+\left(y_{0}-\bar{y}_{0}\right) \log \frac{(a-d v)}{y(0)}+\left(z_{0}-\bar{z}_{0}\right) \log \frac{v}{z(0)}=0
$$

for $v=z$. If we denote the left side of this last equation by $M(v)$, we get

$$
M^{\prime}(v)=\frac{-e\left(x_{0}-\bar{x}_{0}\right)}{b-e v}-\frac{d\left(y_{0}-\bar{y}_{0}\right)}{a-d v}+\frac{z_{0}-\bar{z}_{0}}{v} .
$$

If $z_{0}-\bar{z}_{0}>0$, then $x_{0}<\bar{x}_{0}$, and $y_{0}<\bar{y}_{0}$; recall that $\left(x_{0}, y_{0}, z_{0}\right)$ and $\left(\bar{x}_{0}, \bar{y}_{0}, \bar{z}_{0}\right)$ are distinct points on the open line segment from $(c / d, 0, a / d)$ to $(b, a, 0)$. So, in this case, $M^{\prime}(v)>0$ for $0<v<a / d$. This shows that $M(v)=0$ has at most one solution. Thus, the solutions

$$
v=(b-x(0)) / e=(a-y(0)) / d
$$

and

$$
v=(b-x) / e=(a-y) / d
$$

coincide, and so $x=x(0), y=y(0), z=z(0)$. But this contradicts the fact that $(x(0), y(0), z(0))$ is not a critical point of $(1)$, and so $(x, y, z)$ cannot be one. In case $z_{0}-\bar{z}_{0}<0$, we have $x_{0}-\bar{x}_{0}>0$ and $y_{0}-\bar{y}_{0}>0$, and a similar argument shows that again $(x, y, z)$ cannot be a critical point of (1).

Since $S \cap \bar{S}$ does not contain critical points of (1), we can conclude that the orbit of $(x(t), y(t), z(t))$ is the entire simple closed curve $S \cap \bar{S}$ and that this solution is periodic.

If $(x(0), y(0), z(0))$ is a critical point of $(1)$, there is clearly nothing more to prove, and our theorem follows.

In case the predator interaction rates are not equal in (1), i.e., if the $e$ in the third equation in (1) is replaced by $e_{1}>e$, it follows easily, using methods similar to those in the proof of Case 1 of the theorem, that $(c / d, 0, a / d)$ is again a reachable critical point, and if $\left(e_{1}-e\right) a+b d>a e+c$, then all solutions of (1) satisfy $\left(x(t)-x_{0}(t)\right)^{2}+$ $y^{2}(t)+\left(z(t)-z_{0}(t)\right)^{2} \rightarrow 0$ as $t \rightarrow \infty$, where $\left(x_{0}(t), z_{0}(t)\right)$ is periodic and may reduce to $(c / d, a / d)$; we omit the details. 
We conclude our paper by stating some results for systems similar to (1). These results follow using methods similar to those used in the previous work.

First, consider the system

$$
\begin{aligned}
& x^{\prime}=a x-x y-d x z-\alpha x^{2}, \\
& y^{\prime}=-b x+x y, \\
& z^{\prime}=-c y+d x y,
\end{aligned}
$$

with no predator interaction and with a carrying capacity for the prey. Here all the constants $a, b, c, d, \alpha$ are positive.

The critical points of $(12)$ are $(0,0,0),(a / \alpha, 0,0),(b, a-\alpha b, 0)$, and $(c / d, 0,(a d-$ $\left.\alpha c) / d^{2}\right)$. Clearly the first two are reachable; if (i) $a>\alpha b$, the third is, and if (ii) $a d>\alpha c$, the fourth is. It is again easy to see that $(0,0,0)$ is unstable.

Theorem 2. If (i) $a>\alpha b$ and $c>b d$, then for any solution $(x(t), y(t), z(t))$ of (12), $x(t) \rightarrow b, y(t) \rightarrow a-\alpha b, z(t) \rightarrow 0$ as $t \rightarrow \infty$. If (ii) $a d>\alpha c$ and $c<b d$, then for any solution of (12), $x(t) \rightarrow c / d, y(t) \rightarrow 0$, and $z(t) \rightarrow(a d-\alpha c) / d^{2}$ as $t \rightarrow \infty$. If (iii) $b d>c>a d / \alpha$, then for any solution of (12), $x(t) \rightarrow a / \alpha, y(t) \rightarrow 0, z(t) \rightarrow 0$ as $t \rightarrow \infty$.

We note that condition (iii) $b d>c>a d / \alpha$ rules out case (ii), i.e., the reachability of the third critical point, and since it also implies $a<\alpha b$, it rules out case (i), i.e., the reachability of the fourth.

Finally, we consider the system

$$
\begin{aligned}
& x^{\prime}=a x-x y-d x z, \\
& y^{\prime}=-b y+x y+e y z, \\
& z^{\prime}=c z+x z-e y z,
\end{aligned}
$$

where $a, b, c, d, e$ are positive constants with $a e \neq b d+c$. Here the model has a positive birthrate for the $z$-predator as well as predator interaction.

Here, we have reachable critical points $(0,0,0),(b, a, 0)$, and $(0, c / e, b / e)$.

Theorem 3. If $a e>b d+c$, then $(b, a, 0)$ is stable and all solutions of (13) satisfy $(x(t), y(t), z(t)) \rightarrow\left(x_{0}(t), y_{0}(t), 0\right)$ as $t \rightarrow \infty$, where $\left(x_{0}(t), y_{0}(t)\right)$ is periodic.

If $a e<b d+c$, then $(0, c / e, b / e)$ is stable and all solutions of (13) satisfy $(x(t)$, $y(t), z(t)) \rightarrow\left(0, y_{0}(t), z_{0}(t)\right)$ as $t \rightarrow \infty$, where $\left(y_{0}(t), z_{0}(t)\right)$ is periodic

It is clear that if the $y$-predator does not prey on the $z$-predator, i.e., if $e=0$, then $z(t) \rightarrow \infty$ as $t \rightarrow \infty$, i.e., all solutions are unbounded for $t \geq 0$.

For more general systems modeling a prey and two predators, cf. [1-4]. These papers are mainly concerned with conditions under which the predators persist, i.e., their populations do not approach zero as $t \rightarrow \infty$. Some of these more general systems allow the predation rates, in our case the constants $d$ and $e$, to depend on the size of the prey population $x$. While the conditions given for those more general systems for the persistence of both predators are not as critical as the one given in our theorem, they do not seem to imply the existence of periodic solutions, as in Case 3 of our theorem.

An interesting open question arising for the case where the hypotheses of Thereom 1 hold, the so-called noncritical case, is whether given any periodic solution of (1) 
in either of the coordinate planes, there exists a unique solution which approaches it orbitally as $t \rightarrow \infty$ from the positive orthant $x>0, y>0, z>0$.

\section{References}

1. R. A. Armstrong and R. McGehee, Some mathematical problems concerning the ecological principle of competitive exclusion, J. Diff. Eqn. 23 (1977), 30-52.

2. Coexistence of two competitors for one resource, J. Theo. Biol. 56 (1976), 499-502.

3. H. I. Freedman and P. Waltman, Mathematical analysis of some three-species food chain models, Math. Biosc. 33 (1977), 259-276.

4. __ Persistence in a model of three interacting predator-prey populations, Math. Biosc. 68 (1984), 213-231.

5. T. Yoshizawa, Stability Theory by Liapunov's Second Method, Math. Soc. of Japan 9 (1966).

Iowa State University, Ames, IA 50011, U.S.A. 